\title{
Performance Evaluation for V-Blast MIMO Systems under Various Modulation Schemes Using Ricean Channel
}

\author{
Gurpreet Singh $^{1}$, Rahul Vij ${ }^{2}$ and Priyanka Mishra ${ }^{3}$ \\ ${ }^{I}$ (Department of Electronics and Communication, Jaypee University of Information Technology) \\ ${ }^{2}$ (Department of Electronics and Communication, Jaypee University of Information Technology) \\ ${ }^{3}$ (Department of Electronics and Communication, Jaypee University of Information Technology)
}

\begin{abstract}
Wireless communication using Multiple Input Multiple Output (MIMO) systems increases spectral efficiency for a given total transmits power. Wireless communication technology has shown that the application of multiple antennas at both transmitter and receiver sides improve the possibility of high data rates through data rates through multiplexing or to improve performance through diversity compared to single antenna systems. In this article, we studied the BER performance of Vertical Bells Lab Layered Space Time Architecture (V-BLAST) [2] Spatial Multiplexing Technique with various decoding techniques like Maximum Likelihood $(M L)$, Minimum Mean Square Error (MMSE), Minimum Mean Square Error + Ordered Serial Interference Cancellation (MMSE+OSIC), MMSE, Zero Forcing, Zero Forcing + Ordered Serial Interference Cancellation $(Z F+O S I C)$ by using different modulation techniques such as BPSK, QPSK, 16-QAM in independent, identically distributed (i.i.d) flat fading channel. In this also we will consider a point to point MIMO communications with ' $N$ ' transmitting antennas and ' $M$ ' receiving antennas $(M \geq N)$. In this article we will compared a different detection techniques with different modulation techniques and finally we will concluded that Maximum Likelihood (V-BLAST ML) decoding technique using BPSK modulation scheme gives better result, QPSK modulation gives almost similar results as BPSK and also we concluded that BER performance of 16-QAM Modulation scheme gives worst result than other modulation techniques in Ricean Channel. Finally we will conclude that ML-VBLAST decoding technique gives the better performance than other decoding techniques using BPSK modulation. Further simulation results for BPSK modulation with only ML decoding technique using various antennas at input and output using rician channel. In this we got more optimal result for $1 \times 4$ antennas for V-BLAST system in rician fading channel.
\end{abstract}

Keywords: - Binary Phase Shift Key (BPSK), Bit Error Rate (BER), Multiple input multiple output (MIMO), Minimum Mean-Squared-Error (MMSE-V-BLAST), Maximum Likelihood (ML), Ordered Serial Interference Cancellation (OSIC), Vertical Bell Laboratories Layered Space-Time (V-BLAST) and Zero-Forcing V-BLAST (ZF--BLAST)

\section{INTRODUCTION}

Wireless communication system with multi-antenna arrays has been a field of intensive research on the last years [14]. The use of multiple antennas at both the transmitter and the receiver sides can drastically improve the channel capacity and data rate [12]. The study of the performance limits of MIMO system [1] becomes very important since it will give lot ideas in understanding and designing the practical MIMO systems [4]. Vertical-Bell Laboratories Layered Space-Time (V-BLAST) Architecture and first practical implementation of this architecture on MIMO wireless communications to demonstrate a spectral efficiency as high as 40bits/s/Hz in real time in the laboratory [3]. Many schemes have been proposed to explode the high spectral efficiency of MIMO channels, among which V-BLAST [3] is relatively simple and easy to implement and can achieve a large spectral efficiency. In V-BLAST [2] at the transmitter de-multiplexes the input data streams into ' $n$ ' independent sub-streams, which are transmitted in parallel over the ' $n$ ' transmitting antennas. At the receiver end, antennas receive the sub-streams, which are mixed and superimposed by noise. Detection process [2] mainly involves three operations: Interference Suppression (nulling), interference cancellation (Subtraction) and Optimal Ordering. The interference nulling process is carried out by projecting the received signal into the null subspace spanned by the interfering signals. This process is done by Gramm-Schmidt Orthogonalization procedure that converts the set of linearly independent vectors into orthogonal set of vectors. Then the symbol is detected. The interference cancellation process is done by subtracting the detected symbols from the received vectors. The optimal Ordering is the last process that ensures the detected symbol has highest Signal to noise ratio (SNR). So, V-BLAST algorithm [3] integrates both linear and non-linear algorithms presented in the interference nulling and interference cancellation respectively. In an independent, identically distributed (i.i.d) Flat fading Ricean channel [5] with ' $\mathrm{N}$ ' transmitting antennas and ' $\mathrm{M}$ ' receiving antennas In this we will considered receiving antennas are greater than or equal to transmitting antennas $(M \geq N)$, the first detected substream has a diversity gain of only $\mathrm{M}-\mathrm{N}+1[9]$. 


\section{CHANNEL MODEL}

Let us consider a communication system with ' $N$ ' number of transmitting antennas and ' $M$ ' number of receiving antennas in an i.i.d Ricean Flat Fading channel [5] shown in Fig. 1.

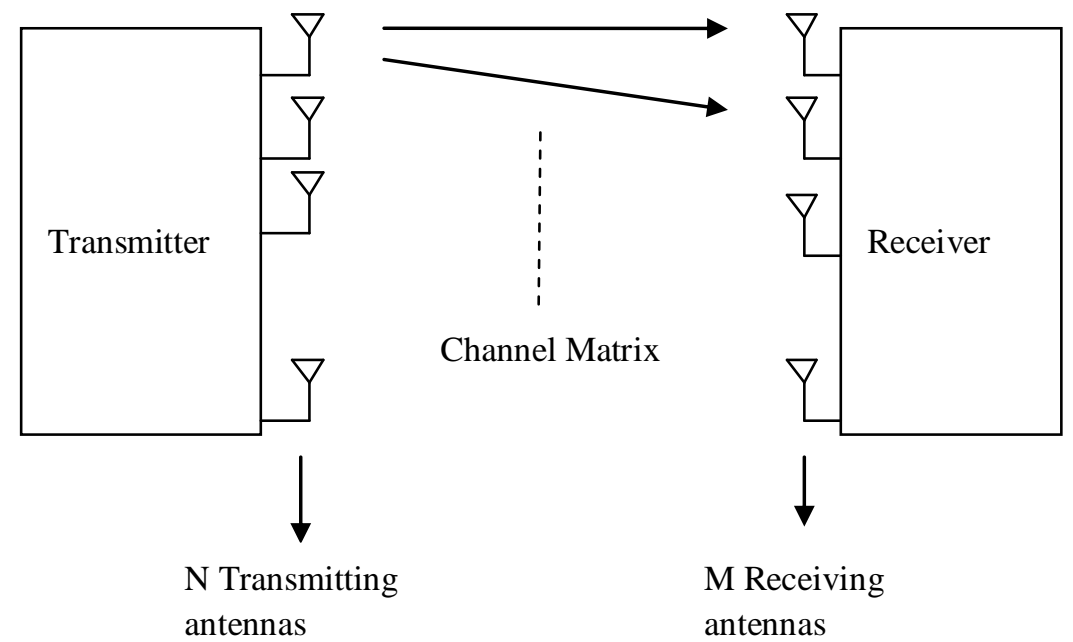

Fig.1 MIMO Block Diagram

The sampled baseband representation of signal is given by

$$
\mathrm{y}=\mathrm{Hx}+\mathrm{n}
$$

And the complex baseband representation of signal [15 ] is given by

$$
y=\sqrt{\frac{P}{M}} H x+n
$$

where $y \in C^{N \times 1}$ is the received signal vector, $x \in C^{M \times 1}$ is the transmitted signal vector with zero mean and unit variance, $\mathrm{P}$ is the total transmit power, $H \in C^{N \times M}$ is the channel response matrix with possibly correlated fading coefficients. In order to access the performance of V-BLAST in correlated channel, we adopted a correlation-based channel model which is expressed as

$$
H \sim R_{R x}^{\frac{1}{2}} H_{w}\left(R_{T x}^{1 \backslash 2}\right)^{T}
$$

where $\mathrm{x} \sim \mathrm{y}$ denotes that $\mathrm{x}$ and $\mathrm{y}$ are identical in distribution, $R_{R x}$ and $T_{T x}$ are the normal correlation distribution matrices at the $\mathrm{Rx}$ and transmitter (Tx) respectively, and $H_{W} \in C^{N \times M}$ contains i.i.d complex Gaussian entries with zero mean and unit variance.

\section{RICEAN FADING CHANNEL}

In practice, the behavior of $\mathrm{H}$ can significantly deviate from $\mathrm{H}_{\mathrm{w}}$ due to a combination of inadequate antenna spacing and/or inadequate scattering leading to spatial fading correlation. Furthermore, the presence of a fixed (possibly line-of-sight or LOS) component in the channel will result in Ricean fading [5].

In the presence of an LOS component between the transmitter and the receiver, the MIMO channel may be modeled as the sum of a fixed component and a fading component and given by following equation

$$
H=\sqrt{\frac{k}{1+k}} \bar{H}+\sqrt{\frac{k}{1+k}} H_{w}
$$

$\sqrt{\frac{\mathrm{k}}{1+\mathrm{k}}} \overline{\mathrm{H}}=\mathrm{E}[\mathrm{H}]$ is the LOS component of the channel.

$\sqrt{\frac{\mathrm{k}}{1+\mathrm{k}}} \mathrm{H}_{\mathrm{w}}$ is the fading component. 
$k \geq 0$ in equation is the Ricean $k$-factor of the channel and is defined as ratio of the power in the LOS component of the channel to the power in the fading component. When $\mathrm{k}=0$, we have pure Rayleigh fading channel. At the other extreme $\mathrm{k}=\infty$ corresponds to a non-fading channel. In general, real-world MIMO channels will exhibit some combination of Ricean fading [5] and spatial fading correlation. With appropriate knowledge of the MIMO channel [1] at the transmitter, the signalling strategy can be appropriately adapted to meet performance requirements. The channel state information could be complete (i.e., the precise channel realization) or partial (i.e., knowledge of the spatial correlation, K-factor, etc.).

\section{SYSTEM MODEL}

A high-level block diagram of a V-BLAST system [2] is shown in
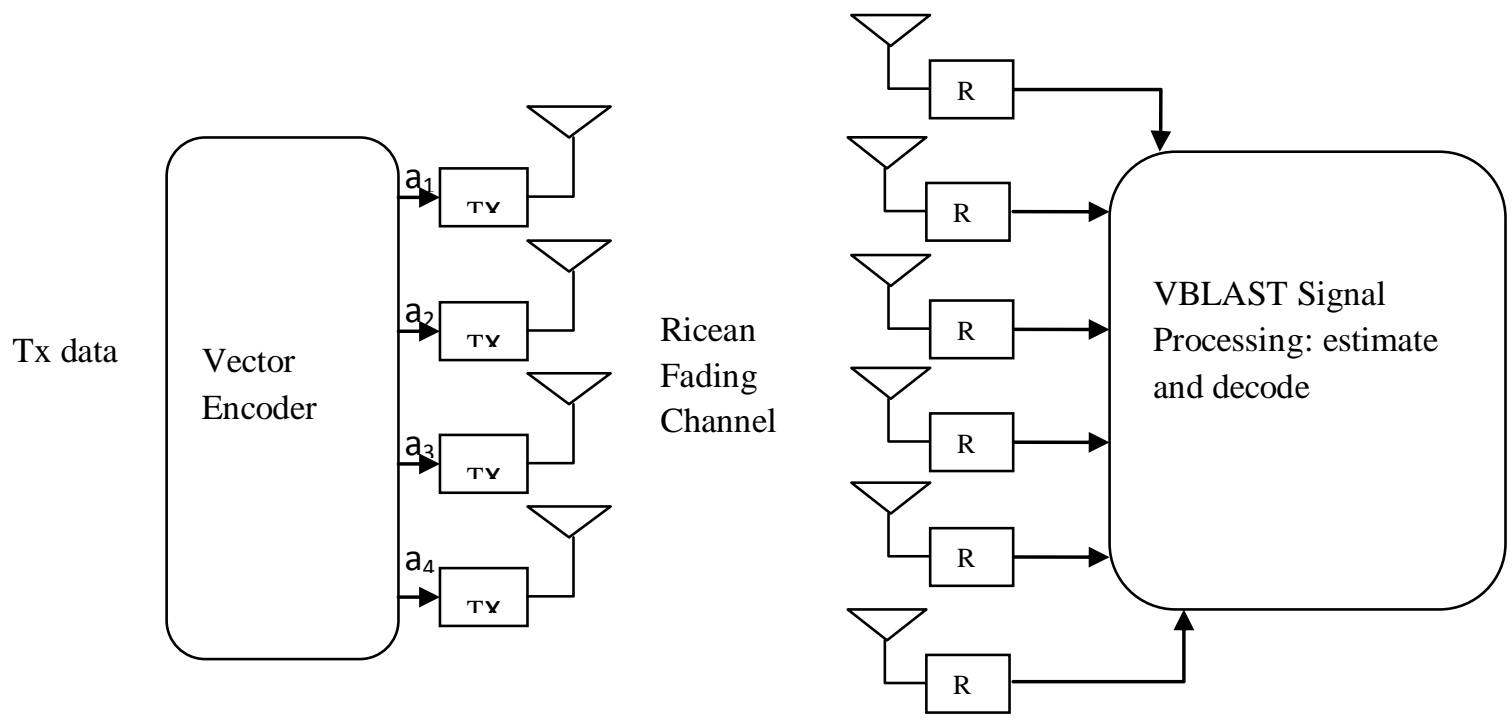

Fig.2 V-BLAST MIMO System Model

\subsection{ENCODER}

A single data stream is de-multiplexed into $\mathrm{m}$ sub-streams, and each sub-stream is then encoded into symbols and fed to its respective transmitter. Transmitters $1-\mathrm{m}$ operate co-channel at symbol rate $1 / \mathrm{T}$ symbols/sec, with synchronized symbol timing. The power launched by each transmitter is proportional to $1 / \mathrm{m}$ so that the total radiated power is constant and independent of ' $\mathrm{m}$ '. At a certain symbol instant, the output of the transmission antenna array is a vector

$$
a=\left[a_{1}, a_{2}, a_{3} \ldots . a_{m}\right]^{T}
$$

\subsection{DECODER}

The decoder needs to demodulate the symbols on the received vector. If channel encoding is used, then the demodulated symbols need to be buffered until the whole block can be decoded. Otherwise, the demodulation can be done immediately.

\section{DETECTION OF V-BLAST SYSTEM}

Detection process mainly involves three operations: Interference Suppression (nulling), interference cancellation (Subtraction) and Optimal Ordering [8]

\subsection{SUCCESSIVE INTERFERENCE CANCELLATION}

At stage $\mathrm{n}$ of the algorithm, when $c_{n}$ is being detected, symbols $c_{1}, c_{2}, \ldots, c_{n-1}$ have been already detected. Let us assume a perfect decoder, that is the decoded symbols $\widehat{c_{1}}, \widehat{c_{2}}, \ldots \ldots, \hat{c}_{n-1}$ are the same as the transmitted symbols $c_{1}, c_{2}, \ldots, c_{n-1}$.

One can subtract $\sum_{i=1}^{n-1} c_{i} H_{i}$ from the received vector $\mathrm{r}$ to derive an equation that relates remaining undetected symbols to the received vector: 


$$
\begin{gathered}
r_{n}=r-\sum_{i=1}^{n-1} c_{i} H_{i}+N, \\
r_{n}=\sum_{i=n}^{N} c_{i} H_{i}+N, \quad n=1,2 \ldots . N-1
\end{gathered}
$$

In fact, by using induction in addition to the convention $r_{1}=r$, one can show that

$$
r_{n+1}=r_{n}-c_{n} H_{n}, \quad n=1,2,3 \ldots . . N-1
$$

Therefore, at the $n^{\text {th }}$ stage of the algorithm after detecting the nth symbol as $\hat{c}_{n}$, its effect is canceled from the equations by

$$
r_{n+1}=r_{n}+\hat{c}_{n} H_{n}
$$

This interference cancelation is conceptually similar to DFE [9].

\subsection{NULLING}

The interference nulling process is carried out by projecting the received signal into the null subspace spanned by the interfering signals. This process is done by Gramm-Schmidt Orthogonalization procedure that converts the set of linearly independent vectors into orthogonal set of vectors [13]

\subsubsection{ZERO FORCING INTERFERENCE BULLING}

Using zero-forcing [15] for interference nulling is common in practice. First, let us assume perfect detection of symbols as in $e q^{n}$ (6) We would like to separate the term $c_{n} H_{n}$ from $r_{n}$. This can be done through multiplying $r_{n}$ by an $\mathrm{M} \times 1$ vector $W_{n}$ that is orthogonal to interference vectors $H_{n+1}, H_{n+2}, \ldots . H_{N}$ but not orthogonal to $H_{n}$.In other words, $W_{n}$ should be such that

$$
\begin{gathered}
H_{i} \cdot W_{n}=0, \quad i=n+1, n+2 \ldots \ldots . N \\
H_{n} . W_{n}=1
\end{gathered}
$$

$W_{n}=$ Zero-Forcing Nulling vector with minimum norm.

Such a vector is uniquely calculated from the channel matrix $\mathrm{H}$. To calculate $W_{n}$ from $\mathrm{H}$, for $\mathrm{M} \geq \mathrm{N}$ first we should replace the rows $1,2 \ldots, \mathrm{n}-1$ of $\mathrm{H}$ by zero.

Let us denote the resulting matrix by $Z$. Then, $W_{n}$ is the nth column of $Z^{+}$the Moore-Penrose generalized inverse, pseudo-inverse, of Z [10]

Using the error-free detection formula for $r^{n}$ in (6) and $w^{n}$ in (10), we have

$$
r_{n} W_{n}=c_{n}+N W_{n}
$$

The noise in (11) is still Gaussian and the symbol $c_{n}$ can be easily decoded. The decoded symbol $\hat{c}_{n}$ is the closest constellation point to $r_{n} . W_{n}$. The noise enhancing factor using (3.11) is

We know that zero forcing is given by

$$
\begin{gathered}
E\left[\left(N \cdot W_{n}\right)^{H} \cdot N \cdot W_{n}\right]=W_{n}^{H} \cdot E\left[N^{H} \cdot N\right] W_{n} \\
=N_{0}\left\|W_{n}\right\|^{2}
\end{gathered}
$$

$$
W_{Z F}=\left(H^{*} H\right) H
$$

Comparing (13) with (14) demonstrates why adding an interference cancelation step improves the performance. Using the combination of canceling and nulling in a ZF-DFE [8] structure enhances the noise by a factor of $\|W n\|^{2}$. Vector $W_{n}$ is orthogonal to $\mathrm{N}-\mathrm{n}$ rows of the channel matrix $\mathrm{H}$. On the other hand, using a pure interference nulling method like ZF, the corresponding vector that detects the nth symbol, the $n^{\text {th }}$ column of the pseudo-inverse, is orthogonal to $\mathrm{N}-1$ rows of the channel matrix H. Using the Cauchy-Schwartz inequality [10], it can be shown that the norm of a vector is larger if it has to be orthogonal to a greater number of rows. Therefore, the enhancing factor for the case of nulling alone, $\mathrm{ZF}$, is more than that of the canceling and nulling, ZF-DFE [9] 


\subsubsection{MMSE-INTERFERENCE NULLING}

Another approach for interference nulling is MMSE [15]. Let us assume that the trans-mitted vector is a zero-mean random vector that is uncorrelated to the noise. Considering the received vector $r$ in (5) as a noisy observation of the input $\mathrm{C}$, the linear least-mean-squares estimator of $\mathrm{C}$ is

$$
M=H^{H} \cdot\left(\frac{I^{N}}{\gamma}+H \cdot H^{H}\right)^{-1}
$$

Note that in the nth stage of the algorithm, the effects of $c_{1}, c_{2}, \ldots, c_{n-1}$ have been canceled. Therefore, similar to the ZF nulling, to calculate $c_{n}$, first we should replace the rows $1,2, \ldots, n-1$ of $\mathrm{H}$ by zero. Let us denote the resulting matrix by $\mathrm{Z}$ as we did in the $\mathrm{ZF}$ case. Now, to find the best estimate of the nth symbol, that is $\hat{c}_{n}$, we replace $\mathrm{H}$ with $\mathrm{Z}$ in (16) to calculate the best linear MMSE estimator at stage $\mathrm{n}$ as

$$
M=Z^{H} \cdot\left(\frac{I^{N}}{\gamma}+Z \cdot Z^{H}\right)^{-1}
$$

Then, the nth column of M, denoted by $M_{n}$ is utilized as the MMSE nulling vector for the $n^{\text {th }}$ symbol. In other words, the decoded symbol $\hat{c}_{n}$ is the closest constellation point to $r_{n} \cdot M_{n}$

\subsection{OPTIMAL ORDERING}

One approach to a lower complexity design of the receiver is to use a "divide-and-conquer" strategy instead of decoding all symbols jointly. First, the algorithm decodes the strongest symbol. Then, canceling the effects of this strongest symbol from all received signals, the algorithm detects the next strongest symbol. The algorithm continues by canceling the effects of the detected symbol and the decoding of the next strongest symbol until all symbols are detected. The optimal detection order is from the strongest symbol to the weakest one. This is the original decoding algorithm of V-BLAST preset [3]. It only works if the number of receive antennas is more than the number of transmit antennas, that is $\mathrm{M} \geq \mathrm{N}$.

In decoding the first symbol, the interference from all other symbols is considered as noise. After finding the best candidate for the first symbol, the effects of this symbol in all of the receiver equations are canceled. Then, the second symbol is detected from the new sets of equations. The effects of the second detected symbol are canceled next to derive a new set of equations. The process continues until all symbols are detected. Of course, the order in which the symbols are detected will impact the final solution.

\section{DECODERS OF V-BLAST SYSTEM}

There are several decoders [14] used in V-BLAST system [2] which are explained bellow one by one.

\subsection{MAXIMUM LIKELIHOOD}

The ML receiver [7] performs optimum vector decoding and is optimal in the sense of minimizing the error probability. ML receiver is a method that compares the received signals with all possible transmitted signal vectors which is modified by channel matrix $\mathrm{H}$ and estimates transmit symbol vector $\widehat{\mathrm{C}}$ according to the Maximum Likelihood principle [7], which is shown as:

$$
\widehat{\mathrm{C}}=\underset{\widehat{\mathrm{C}}}{\operatorname{argmin}} \llbracket \mathrm{r}-\mathrm{C}^{\prime} \mathrm{H} \rrbracket_{\mathrm{F}}^{2}
$$

where $_{\mathrm{F}}$ is the Frobenius norm. Expanding the cost function using Frobenius norm given by

$$
\begin{gathered}
\widehat{\mathrm{C}}=\underset{\widehat{\mathrm{C}}}{\operatorname{argmin}}\left[\operatorname{Tr}\left[\left(\mathrm{r}-\mathrm{C}^{\prime} \mathrm{H}\right)^{\mathrm{H}} \cdot\left(\mathrm{r} \mathrm{O} 0-\mathrm{C}^{\prime} \mathrm{H}\right)\right]\right\rceil \\
\widehat{\mathrm{C}}=\underset{\widehat{\mathrm{C}}}{\operatorname{argmin}}\left\lceil\operatorname{Tr}\left[\mathrm{r}^{\mathrm{H}} \cdot \mathrm{r}+\mathrm{H}^{\mathrm{H}} \cdot \mathrm{C}^{\prime} \mathrm{H} \cdot \mathrm{C}^{\prime} \cdot \mathrm{H}-\mathrm{H}^{\mathrm{H}} \cdot \mathrm{C}^{\prime} \mathrm{H} \cdot \mathrm{r}-\mathrm{r}^{\mathrm{H}} \cdot \mathrm{C}^{\prime} \cdot \mathrm{H}\right]\right\rceil
\end{gathered}
$$

Considering $\mathrm{r}^{\mathrm{H}} \cdot \mathrm{r}$ is independent of the transmitted codeword so can be rewritten as

$$
\widehat{\mathrm{C}}=\underset{\widehat{\mathrm{C}}}{\operatorname{argmin}}\left\lceil\operatorname{Tr}\left\lceil\mathrm{H}^{\mathrm{H}} \cdot \mathrm{C}^{\prime} \mathrm{H} \cdot \mathrm{C}^{\prime} \cdot \mathrm{H}\right\rceil-2 \cdot \operatorname{Real}\left(\operatorname{Tr}\left[\mathrm{H}^{\mathrm{H}} \cdot \mathrm{C}^{\prime} \mathrm{H} \cdot \mathrm{r}\right]\right)\right\rceil
$$

Equation "(20)" can be rewritten for multiple receivers as shown in

$$
\widehat{\mathrm{C}}=\underset{\widehat{\mathrm{C}}}{\operatorname{argmin}}\left\lceil\sum_{\mathrm{m}=1}^{\mathrm{M}_{\mathrm{R}}}\left[\mathrm{H}_{\mathrm{m}}^{\mathrm{H}} \cdot \mathrm{C}^{\prime} \mathrm{H} \cdot \mathrm{C}^{\prime} \cdot \mathrm{H}_{\mathrm{m}}-2 \cdot \operatorname{Real}\left(\mathrm{H}_{\mathrm{m}}^{\mathrm{H}} \cdot \mathrm{C}^{\prime} \cdot \mathrm{r}_{\mathrm{m}}\right)\right]\right\rceil
$$

where ${ }^{\mathrm{H}}$ is a Hermition operator. We can write the cost function for only one receiving antenna and then added up to achieve for $\mathrm{M}_{\mathrm{R}}$ receiving antenna.

$$
\left.\left\lceil\mathrm{H}_{\mathrm{m}}^{\mathrm{H}} \cdot \mathrm{C}^{\prime} \mathrm{H} \cdot \mathrm{C}^{\prime} \cdot \mathrm{H}_{\mathrm{m}}-2 \cdot \operatorname{Real}\left(\mathrm{H}_{\mathrm{m}}^{\mathrm{H}} \cdot \mathrm{C}^{\prime} \cdot \mathrm{r}_{\mathrm{m}}\right)\right]\right\rceil
$$

where the minimization is performed over all possible transmit estimated vector symbols. Although $\mathrm{ML}$ detection offers optimal error performance, it suffers from complexity issues. 


\subsection{V-BLAST ZERO FORCING DECODER}

Zero Forcing [15] is the linear MIMO technique. The processing takes place at the receiver where, under the assumption that the channel matrix $\mathrm{H}$ is invertible [10], $\mathrm{H}$ is inverted and the transmitted MIMO vector ' $\mathrm{s}$ ' is estimated by

$$
s_{\text {est }}=H^{-1} x
$$

For Zero Forcing, nulling of the "interferers" can be performed by choosing $1 \mathrm{x} \mathrm{N}$ dimensional weight vectors $w^{i}$ (with $\mathrm{i}=1,2 \ldots \ldots . . \mathrm{M}$ ), referred to as nulling vectors, such that

$$
w^{i} h_{p}=\left\{\begin{array}{l}
0, p \neq i \\
1, p=i
\end{array}\right\}
$$

where $\mathrm{h}$ denotes the $\mathrm{p}$-the column of channel matrix $\mathrm{H}$. Let $w^{i}$ be the $\mathrm{i}$-th row of the matrix $\mathrm{W}$, then it follows that

$$
W=H I_{N}
$$

Where $\mathrm{W}$ is the matrix that represents the linear processing of in the receiver. So, by forcing the "interferers" to zero, each desired element of $\mathrm{s}$ can be estimated.

If $\mathrm{H}$ is not square, $\mathrm{W}$ equals the pseudo-inverse of $\mathrm{H}$ [9] denoted by $H^{+}$

$$
W=H^{+}=\left(H^{H} H\right)^{-1} H^{H}
$$

If elements of $H$ are assumed to be i.i.d [10], the pseudo-inverse [9] exists, when $M \geq N$. For $M \leq N$, $H^{H} H$ is singular and its inverse does not exists [9]. When the pseudo-inverse exits, the estimates of s (given by $S_{\text {est }}$ ) can be given by

$$
\begin{gathered}
s_{\text {est }}=W x=\mathrm{H}^{+}=\left(H^{H} H\right)^{-1} H^{H} \mathrm{X} \\
s_{\text {est }}=\mathrm{s}+\left(H^{H} H\right)^{-1} H^{H} \mathrm{n}
\end{gathered}
$$

The big disadvantage of Zero Forcing [13] is that is suffers from noise enhancement. This can readily observed from above equation.

This leads to estimation error and given by following equation

$$
\epsilon=s_{\text {est }}-\mathrm{s}=\left(H^{H} H\right)^{-1} H^{H} \mathrm{n}
$$

The $\mathrm{ZF}$ receiver converts the joint decoding problem into $\mathrm{M}$ single stream decoding problems thereby significantly reducing receiver complexity. This complexity reduction comes, however at the expense of noise enhancement which results in a significant performance degradation.

\subsection{V-BLAST MINIMUM MEAN SQUARE DECODER}

The MMSE [15] receiver suppresses both the interference and noise components, whereas ZF receiver removes only the interference components. This implies that the mean square error between the transmitted symbols and the estimate of the receiver is minimized. Hence MMSE is superior to ZF in the presence of noise. At low SNR, MMSE becomes matched filter and at high SNR, MMSE becomes Zero Forcing (ZF). For MMSEV-BLAST [10], the nulling vector for the i-th layer is

$$
w^{i}=\left[H_{i} H_{i}^{*}+\frac{1}{\operatorname{snr}} I\right]^{-1} h_{i}, \quad i=1,2 \ldots \ldots N
$$

Where $H_{i}=C^{M \times i}$ consists of the first I columns of $\mathrm{H}$. Then the post-processing SNR of the $\mathrm{i}$-th layer is

$$
\rho_{i}^{M M S E}=\frac{\left|h_{i}^{*}\right|^{2}}{w_{i}^{*}\left(H_{i-1} H_{i-1}^{*}+s n r^{-1} I\right) w_{i}}
$$

Inserting (18) into (19), we can simplify via some straight forward calculations that are

$$
\rho_{i}^{M M S E}=h_{i}^{*} C_{i}^{-1} h_{i} \quad \mathrm{i}=1,2 \ldots \ldots . \mathrm{N}
$$

where $C_{i}^{-1}=H_{i-1} H_{i-1}^{*}+s n r^{-1} I$, applying the matrix inversion, we obtain

Inserting (21) into (20) we get

$$
C_{i}^{-1}=\operatorname{snr}\left[\mathrm{I}-H_{i-1} H_{i-1}^{*}+s n r^{-1} I\right]^{-1} \mathrm{H}_{\mathrm{i}-1}^{*}
$$

$$
\begin{aligned}
\rho_{i}^{M M S E}=\operatorname{snr} r_{i}^{*} P & \frac{1}{H_{i-1}} \\
& +\operatorname{snr} r_{i}^{*} H_{i-1}\left[\left(H_{i-1} H_{i-1}^{*}\right)^{-1}-\left(H_{i-1} H_{i-1}^{*}+s n r^{-1} I\right)^{-1}\right] \mathrm{H}_{\mathrm{i}-1}^{*} \mathrm{~h}_{\mathrm{i}} \\
\rho_{i}^{M M S E}=\rho_{\mathrm{i}}^{\mathrm{ZF}} & +\operatorname{snr} h_{i}^{*} H_{i-1}\left[\left(H_{i-1} H_{i-1}^{*}\right)^{-1}-\left(H_{i-1} H_{i-1}^{*}+s n r^{-1} I\right)^{-1}\right] \mathrm{H}_{\mathrm{i}-1}^{*} \mathrm{~h}_{\mathrm{i}}
\end{aligned}
$$


Hence MMSE receiver approaches the ZF receiver and therefore realizes $(\mathrm{N}-\mathrm{M}+1)$ th order diversity [5] for each data stream.

\subsection{ZERO FORCING WITH OSIC DECODER}

OSIC [15] is basically based on subtraction of interference of already detected elements of s from the receiver vector $\mathrm{x}$. This results in a modified receiver vector in which effectively fewer interferers are present. Decoding algorithm consists of basically three steps which are summarizing

1) Compute $H^{+}$, find the minimum squared length row of $H^{+}$, say it is the $\mathrm{p}^{\text {th }}$ and permute it to be last row. Permute columns of $\mathrm{H}$ accordingly.

2) From the estimate of the corresponding elements of s. In case of $\mathrm{ZF}$ :

$$
\left(s_{e s t}\right)_{P}=W^{n} x
$$

Where the weight vector $W^{n}$ equals row $\mathrm{N}^{\mathrm{t}}$ of the permuted $H^{+}$

3) While M-1>0 go back to step 1 , but now with:

$$
H \rightarrow H^{(M-1)}=\left(h_{1} \ldots \ldots . . h_{M-1}\right)
$$

So we can see here with respect to $\mathrm{ZF}$, the ZF with OSIC algorithm introduces extra complexity.

\subsection{MMSE WITH OSIC}

In order to do OSIC with MMSE [15], then the algorithm resulting as follows

Covariance matrix can be written as

$$
\left[\left(s-s_{\text {est }}\right)\left(s-s_{\text {est }}\right)^{H}\right]=\sigma_{n}^{2}\left(\alpha I+H H^{H}\right)^{-1} \equiv \sigma_{n}^{2} P
$$

Covariance matrix of the estimation error $\left(s-s_{\text {est }}\right)$ will be used to determine good ordering for detection.

1) Compute $\mathrm{W}(\mathrm{P}$ is obtained while determining $\mathrm{W})$. Find the smallest diagonal entry of $\mathrm{P}$ and suppose this is the p-th entry. Permute the $\mathrm{p}$-th column of $\mathrm{H}$ to be last column and permute the rows of $\mathrm{W}$ accordingly.

2) From the estimate of the corresponding elements of s. In case of MMSE:

$$
\left(s_{\text {est }}\right)_{P}=W^{M} x
$$

Where the weight vector $W^{M}$ equals row $\mathrm{M}$ (number of transmitting antennas) of the permuted W

3) While M-1>0 go back to step 1, but now with:

$$
H \rightarrow H^{(M-1)}=\left(h_{1} \ldots \ldots \ldots h_{M-1}\right)
$$

So here we can see that we get optimal ordering by using MMSE with OSIC

\section{SIMULATIONS AND RESULTS}

I am used a MATLAB 7.0 for simulation for the Bit Error Rate (BER) Performance of the Vertical Bells Lab Layered Space Time Architecture (VBLAST) System [13]. I simulate the BER performance of VBLAST using various detectors like Maximum Likelihood, MMSE, Zero Forcing, ZF-SIC, and MMSE-SIC [14] in Ricean flat fading channel [5] by using the different modulation techniques like BPSK, QPSK and 16QAM.

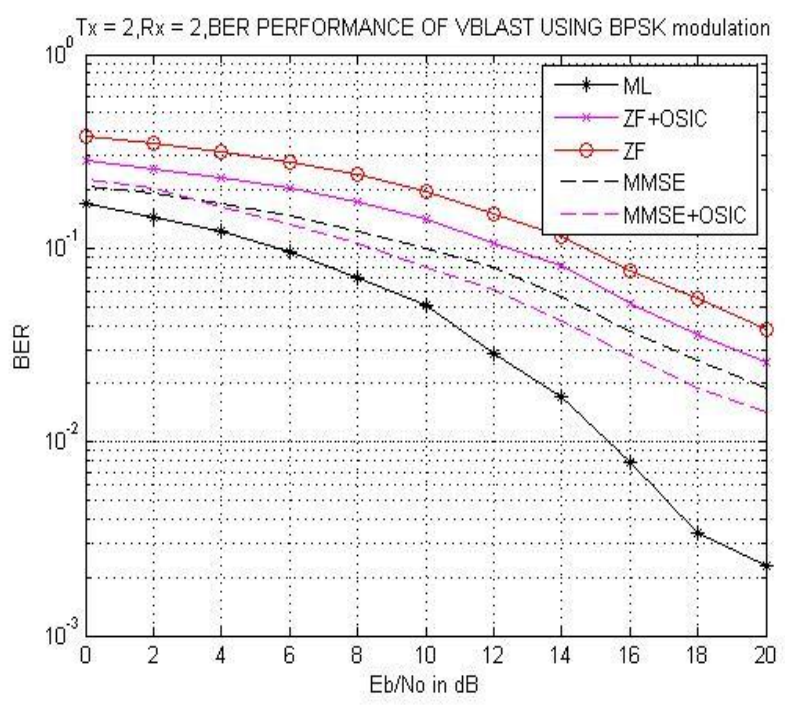

Fig.3: BER for VBLAST using BPSK modulation 


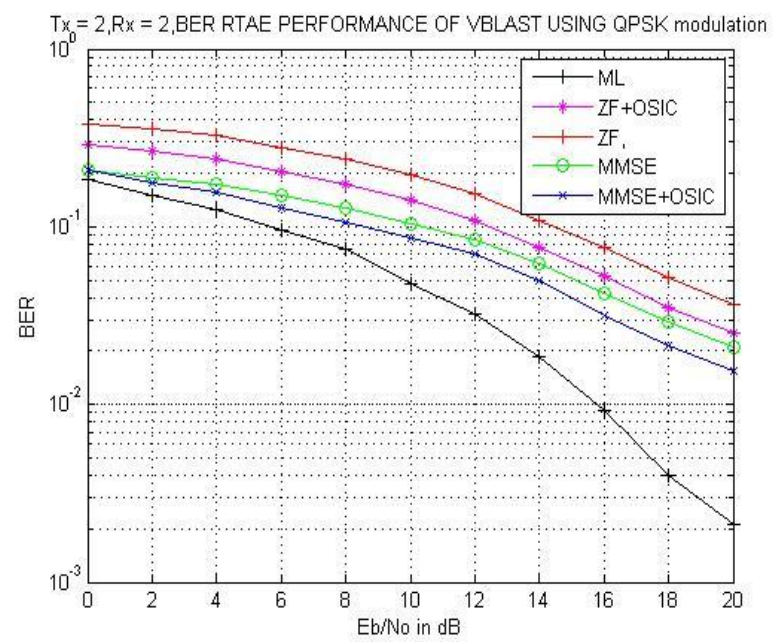

Fig.4: BER for VBLAST using QPSK modulation

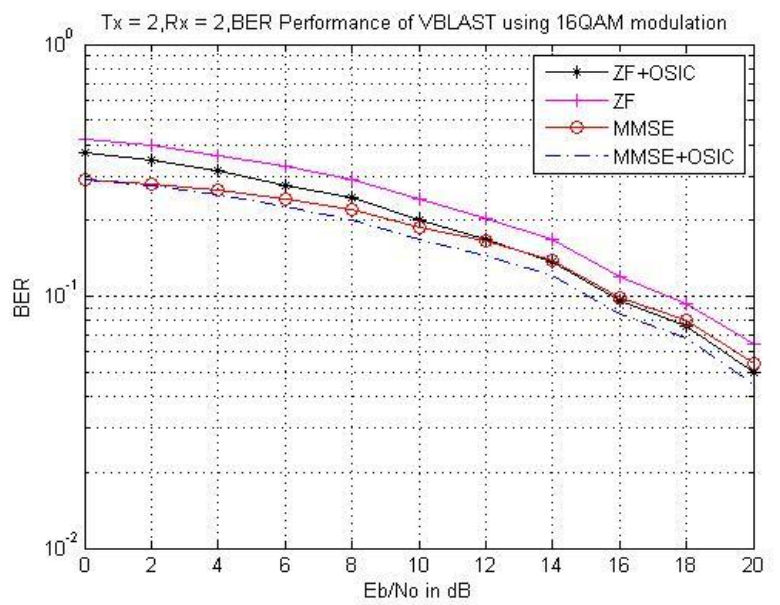

Fig.5: BER for VBLAST using 16-QAM modulation

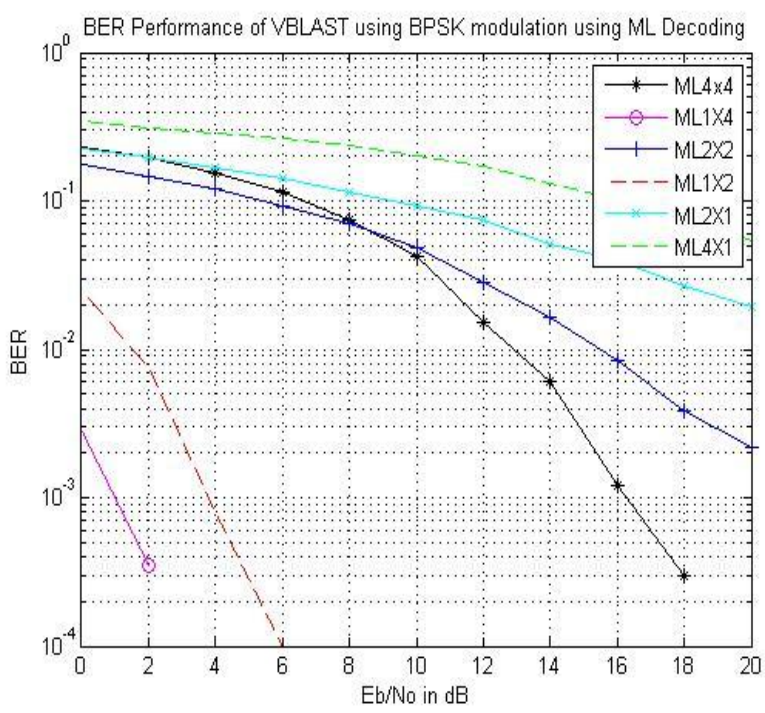


Fig.6: BER for VBLAST using BPSK modulation using ML decoding using different antennas

The above graph Fig.3 is plot between SNR (dB) and BER using BPSK Modulation in Ricean Channel [5]. There is a comparison between the different detectors like Maximum Likelihood (ML), ZF-OSIC, ZF, MMSE and MMSE-OSIC which are used at receiver in V-BLAST System. Here we observed that Maximum Likelihood (ML) have a best performance than other detectors which are used at receiver in V-BLAST System and Zero Forcing (ZF) has a worst performance. If we compare the ZF and ML, performance curve of the two detectors are close to each other at low SNR but the gap gets larger when SNR gets higher. When the SNR gets higher, the post detection of SNR is mainly affected by channel matrix H. If we compare the MMSE-OSIC and $\mathrm{ZF}-\mathrm{OSIC}$, at BER=0.01 there is an approximately $4 \mathrm{~dB}$ difference between these two detectors.

The above graph Fig.4 is plot between SNR (dB) and BER using QPSK Modulation in Ricean Channel. There is a comparison between the different detectors like Maximum Likelihood (ML), ZF-OSIC, ZF, MMSE and MMSE-OSIC which are used at receiver in V-BLAST System. Here we observed that Maximum Likelihood (ML) have a best performance than other detectors which are used at receiver in V-BLAST System and Zero Forcing (ZF) has a worst performance. If we compare the $\mathrm{ZF}$ and ML, performance curve of the two detectors are close to each other at low SNR but the gap gets larger when SNR gets higher. When the SNR gets higher, the post detection of SNR is mainly affected by channel matrix H. If we compare the MMSE-OSIC and $\mathrm{ZF}-\mathrm{OSIC}$, at BER=0.01 there is an approximately $4 \mathrm{~dB}$ difference between these two detectors.

The above graph Fig.5 is plot between SNR (dB) and BER using BPSK Modulation in Ricean Channel. There is a comparison between the different detectors like ZF-OSIC, ZF, MMSE and MMSE-OSIC which are used at receiver in V-BLAST System. Here we observed that MMSE have a best performance than other detectors which are used at receiver in V-BLAST System and Zero Forcing (ZF) has a worst performance. When the SNR gets higher, the post detection of SNR is mainly affected by channel matrix H. If we compare the MMSE-OSIC and ZF-OSIC, at BER=0.01 there is an approximately $1.3 \mathrm{~dB}$ difference between these two detectors.

\section{CONCLUSION}

In this article, we studied MIMO V-BLAST system performance under i.i.d Ricean channel [5]. Further this system is compared with different modulation technique and system gets better result in BPSK modulation and 16-QAM modulation technique gives worst result with different detection technique. Fig. 6 shows the simulation results for BPSK modulation with only ML decoding technique using various antennas at input and output. In this we will more optimal result for 1 x 4 antennas for V-BLAST system.

\section{REFERENCES}

[1] R. U. Nabar A. J. Paulraj, D. A. Gore and H. Bolcskei, "An overview of MIMO communications-a key to gigabit wireless," Proceedings of the IEEE, vol. 92, no. 2, pp. 198-218, Feb. 2004

[2] G.D.Golden, G.J.Foschini, R.A. Valenzuela, and P.W.Wolniasky, "Detection algorithm and initial laboratory results using the VBLAST space-time communication architecture," Electron Lett., vol.35, no.1, pp.1415, 1999.

[3] P.Wolniosky, G.J.Foschini, G.D.Golden and R.A. Valenzuela," V-BLAST: An Architecture for realizing very high data rates over rich scattering wireless channel” URSI International Symposium on Signals, Systems and Electronics, 1998. ISSSE 98, 1998.

[4] A.Paulraj and R.J.Heath, "Characterization of MIMO Channels for Spatial Multiplexing Systems "IEEE International Conference on Communications, vol.2, no.11-14,pp-591-595,June 2001.

[5] R.U. Nabar, H.Boleskei and A.J. Paulraj," Diversity and outage performance in Space Time Block Coded Rician MIMO Channels" IEEE Trans. Wireless Commun. Vol.4, pp.1102-1111, May 2005.

[6] G. J. Foschini, "Layered space-time architecture for wireless communication in a fading environment using multi-element antennas," Bell-Labs Techn. J., pp. 41-59, 1996.

[7] C.Windpassinger and RF.H Fischer, "Low-complexity need-Maximum Likelihood detection and precoding for MIMO systems" in ITW 2003, Paris, France, March 31-April-4, 2003.

[8] M.Varanasi and T.Guess, "Optimum decision feedback multiuser equalization with successive decoding achieves the total capacity of the Gaussian multiple-access channel," Conference Record of the Thirty-First Asilomar Conference on signals, Systems and computers, vol. 2, pp. 1405-1409, Nov-2-5 1997.

[9] G.Ginis and J.M.Cioffi, "On the relationship between V-BLAST and GDFE," IEEE Communications letters, vol. 5, pp. 364-366, September 2001.

[10] A.M.Tulino and S.Verdu, Random Matrix Theory and Wireless Communications. Hanover, MA 02339, USA: now publishers Inc., 2004.

[11] H. El Gamal and A.R. Hammons, "The layered space-time architecture: a new perspective", IEEE Trans. Inform. Theory, vol. 47, pp. 2321-2334, Sept. 2001.

[12] I.E. Telatar, "Capacity of multi-antenna Gaussian channels, "European Transactions on Telecommunications, vol. 10, no.6, pp.585595, November/December 1999.

[13] A. Paulraj, R.Nabar and D.Gore, "Introduction to Space Time Wireless Communications", Cambridge University Press, May 2003.

[14] X.Li, H.Huang, G.J.Foschini, and R.A.Valenzu, "Effects of Iterative Detection and Decoding on the Performance of BLAST", IEEE Global Telecommunications Conference, vol.2, pp.1061-10066, Nov 2000. 
[15] Choi, J, Yu,H., and Lee, Y.H.(2005) "Adaptive MIMO decision Feedback Equalization for Receivers with time varying channels", IEEE transaction on signal processing, vol.55, No. 7, pp.3405-3416

\section{BIOGRAPHY}

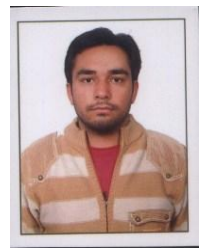

Mr.Gurpreet Singh received an MTECH degree in Electronics and Communication Engineering degree from Jaypee University of Information and Technology, Solan in 2012 and qualified a BTECH degree from Lovely Institutes of Technology in Electronics and Communication Engineering from Lovely Institutes of Technology, Phagwara in 2010 with distinction. Now he is currently working as Assistant Professor in Shaheed Bhagat Singh State Technical Campus, Ferozpur, Punjab. He also presented a paper in national and international conferences and also published a many papers in International Journal. His current research interests in the area of signal processing, MIMO, Wireless Mobile Communication Engineering, high speed digital communications and 4G Wireless Communications.

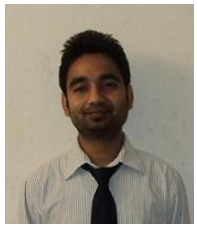

Mr. Rahul Vij received a BTech degree from GGSCET, Talwandi Sabo, Bathinda under PTU, Jalandhar in May, 2008 and qualified an MTech degree from Jaypee University of Information and Technology, Solan in 2012. He worked as a Lecturer in ECE department in Eternal University, Baru Sahib, Distt. Sirmour, Himachal Pradesh. Upto april,2010, he has been associated with the Eternal University, Sirmour, Himachal Pradesh where he is worked as Lecturer in Electronics and Communication Depatment. Now currently he is working as Asst. Professor in ECE department in L.R Institute of engineering and technology, Solan, Himachal Pradesh. His area of intrest in MIMO Technologies, Digital electronics, Analog electronics, semiconductor devices and Wireless Communications

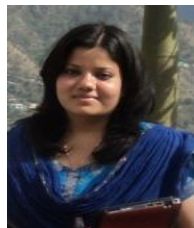

Ms. Priyanka Mishra completed an MTECH degree in Electronics and Communication Engineering degree from Jaypee University of Information Technology, Solan in 2012 and received a B.Tech degree from BBS College of engineering \& technology in 2009. She currently is working as Assistant Professor in Electronics and Communication Engineering department in the United Group of Institutions, Allahabad, Greater Noida. Her area of interest in the Wireless communication, data communication, MIMO technologies and wireless $4 \mathrm{G}$ communication. 